\title{
The learning curve of thoracoscopic surgery in a single surgeon and successful implementation of uniportal approach
}

\author{
Hitoshi Igai^, Mitsuhiro Kamiyoshihara, Shinya Furusawa, Fumi Ohsawa, Tomohiro Yazawa, \\ Natsumi Matsuura
}

Department of General Thoracic Surgery, Japanese Red Cross Maebashi Hospital, Maebashi, Gunma, Japan

Contributions: (I) Conception and design: H Igai; (II) Administrative support: H Igai; (III) Provision of study materials or patients: All authors; (IV) Collection and assembly of data: All authors; (V) Data analysis and interpretation: H Igai; (VI) Manuscript writing: All authors; (VII) Final approval of manuscript: All authors.

Correspondence to: Hitoshi Igai. Department of General Thoracic Surgery, Japanese Red Cross Maebashi Hospital, 389-1 Asakura-cho, Maebashi, Gunma 371-0811, Japan. Email: hitoshiigai@gmail.com.

Background: In some institutions, a recently introduced uniportal approach has replaced the multiportal
approach for thoracoscopic major pulmonary resection. This study investigated the effect of this change on
the surgical learning curve by examining the perioperative results of a single surgeon.
Methods: Between April 2012 and August 2020, 376 patients with primary lung cancer underwent
thoracoscopic lobectomy with ND2a-1/2 lymphadenectomy in the authors' hospital. Surgery was performed
by one of the authors in 189 of these patients, who were thus enrolled in this retrospective study. The
surgeries were classified chronologically into five phases and the operative time, rate of intraoperative massive
bleeding, and rate of postoperative prolonged air leak (PAL) were then compared. The learning curve (i.e.,
operative time) was assessed by Spearman's rank correlation test. The perioperative results achieved with the
uniportal and multiportal approaches were also compared before and after the patients were matched for
their characteristics based on the propensity score. Results: The five phases differed significantly with respect to the operative time and rate of postoperative PAL $(\mathrm{P}<0.0001, \mathrm{P}=0.0061)$. The correlation between operative time and number of consecutive cases was also significant $(\mathrm{r}=-0.579, \mathrm{P}<0.0001)$. Superior results in terms of operative time $(\mathrm{P}<0.0001)$, duration of postoperative drainage $(\mathrm{P}<0.0001)$, and rate of postoperative $\mathrm{PAL}(\mathrm{P}=0.0034)$ were obtained using a uniportal rather than multiportal approach.

Conclusions: The transition from a multiportal to a uniportal approach did not cause a decline in the learning curve of thoracoscopic lobectomy with ND2a-1/2 lymphadenectomy.

Keywords: Uniportal approach; multiportal approach; thoracoscopic lobectomy; learning curve

Submitted Mar 21, 2021. Accepted for publication May 27, 2021.

doi: $10.21037 /$ jtd-21-500

View this article at: https://dx.doi.org/10.21037/jtd-21-500

\section{Introduction}

A uniportal thoracoscopic approach for major pulmonary resection was first reported in 2013 by Gonzalez and colleagues after Rocco and colleagues had firstly reported the wedge resection via uniportal approach in 2004, and has since been gaining worldwide acceptance $(1,2)$. A uniportal thoracoscopic approach is less invasive than a multiportal approach, as it requires fewer skin incisions. The efficacy

\footnotetext{
$\wedge$ ORCID: 0000-0002-5916-8209.
} 


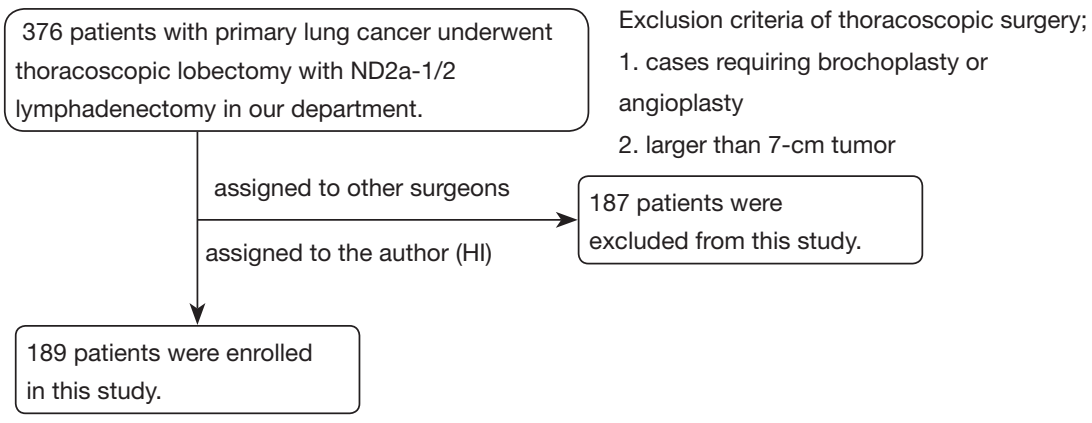

Figure 1 The patient enrolment process.

of a uniportal thoracoscopic approach for major pulmonary resection has been demonstrated in several reports (3-5). Al-Ameri et al. found that uniportal and multiportal approaches for thoracoscopic lobectomy were equivalent in terms of feasibility and safety, but the former has an additional advantage of faster postoperative recovery (3). Bourdages-Pageau et al. reported a significantly shorter operative time, shorter-duration postoperative chest tube drainage and hospitalization periods, and decreased intraoperative bleeding using a uniportal rather than multiportal approach. However, they noted that the former was performed likely to be performed by a highly experienced surgeon (4).

However, a uniportal thoracoscopic approach, while less invasive, is technically more difficult because, unlike with a multiportal approach, the surgical instruments are simultaneously inserted via a single skin incision, and may interfere with each other during surgery. This has raised doubts among thoracic surgeons regarding the safety and quality of surgery performed with a uniportal thoracoscopic approach, and thus to hesitancy in its adoption.

One of the authors (HI) began performing thoracoscopic major pulmonary resections via three or four ports in April 2012, but switched to the uniportal approach in February 2019. Initially, the author was concerned about the learning curve of thoracoscopic surgery, but later considered that this could be reduced by adopting surgical techniques suitable to a uniportal approach. Thus, this study investigated the learning curve of thoracoscopic major pulmonary resection during the transition from a multiportal to a uniportal approach, based on the perioperative results of surgeries performed by a single surgeon.

We present the following article in accordance with the STROBE reporting checklist (available at https://dx.doi. org/10.21037/jtd-21-500).

\section{Methods}

The study was conducted in accordance with the Declaration of Helsinki (as revised in 2013). The study was approved by institutional ethics board of Japanese Red Cross Maebashi Hospital (NO.: 2021-13) and individual consent for this retrospective analysis was waived.

Between April 2012 and August 2020, 376 patients with primary lung cancer underwent thoracoscopic lobectomy with ND2a-1/2 lymphadenectomy in the author's hospital. Surgery was performed by one of the authors (HI) in 189 patients, who were enrolled retrospectively in this study. Patient data was pulled from individual charts by the author (HI). Before the study period, the author (HI) adopted mini-thoracotomy approach as minimally invasive thoracic surgery. The skin incision was 5 to $8 \mathrm{~cm}$ via the approach. The author (HI) has the experience of performing about 150 pulmonary lobectomies via mini-thoracotomy approach. Figure 1 shows the patient enrollment process. Patients requiring bronchoplasty or angioplasty, or with a tumor $>7 \mathrm{~cm}$ in diameter, were not eligible for thoracoscopic surgery.

Totally thoracoscopic major pulmonary resection was introduced in the authors' hospital in April 2012. The procedure was performed via three or four ports until February 2019, when the surgeon-author switched to uniportal thoracoscopic major pulmonary resection. Initially, to ensure the safety of the operation, the uniportal approach was limited to cT1N0 cases, a strategy that also allowed the surgical team to become familiar with this less invasive procedure. In all other major pulmonary resections, the use of a multiportal approach continued, until December 2019, when all patients with primary lung cancer were treated using the uniportal approach. 


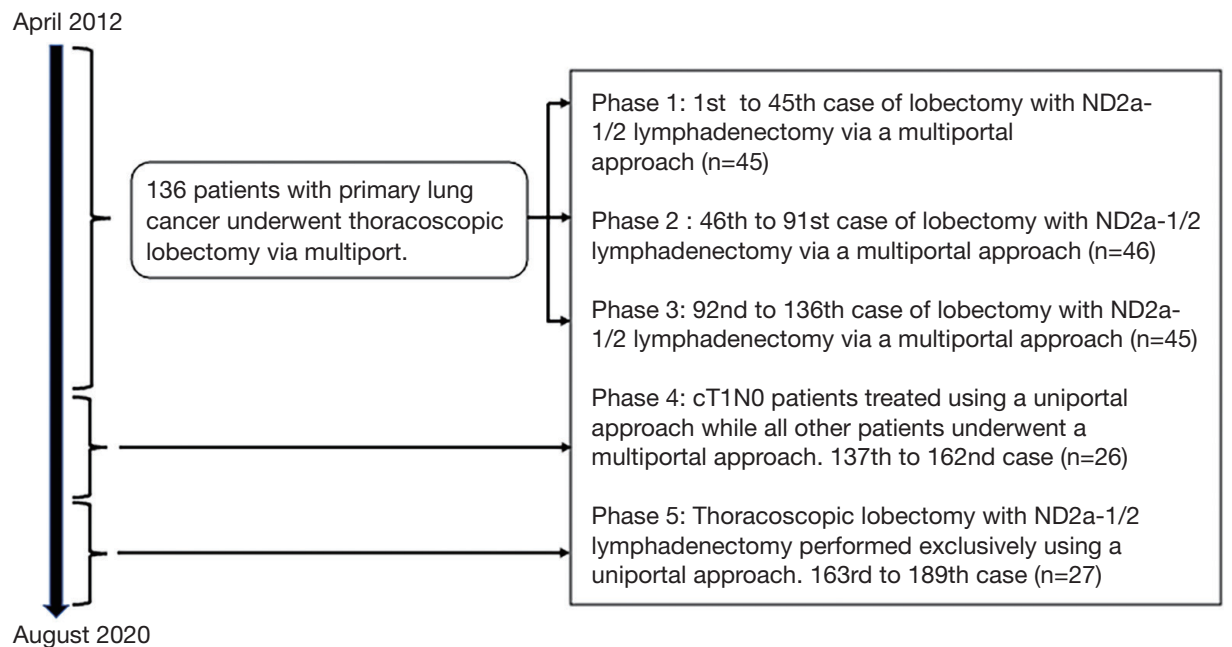

Figure 2 Patient classification.

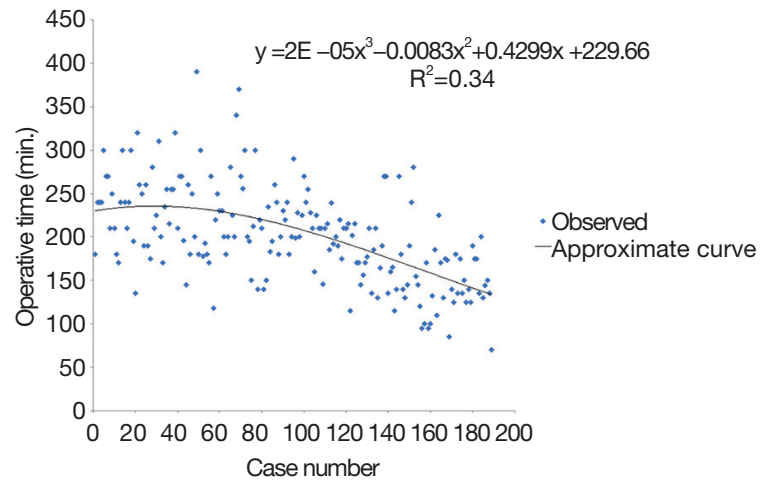

Figure 3 Correlation between the operative time and number of consecutive cases $(n=189)$.

\section{Evaluation of the learning curve}

To evaluate the learning curve of thoracoscopic lobectomy, the surgical period was first divided into three phases: multiportal $(n=136)$, mixed $(n=26)$, and uniportal $(n=27)$. Subsequently, patients who underwent surgery during the multiportal period were divided into three groups: first $(n=45)$, second $(n=46)$, and third $(n=45)$. Therefore, five surgical phases were ultimately examined in this study:

* Phase 1: 1st to 45 th case of thoracoscopic lobectomy with ND2a-1/2 lymphadenectomy via a multiportal approach $(\mathrm{n}=45)$;

* Phase 2: 46th to 91st case of thoracoscopic lobectomy with ND2a-1/2 lymphadenectomy via a multiportal approach $(\mathrm{n}=46)$;
* Phase 3: 92nd to 136th case of thoracoscopic lobectomy with ND2a-1/2 lymphadenectomy via a multiportal approach $(\mathrm{n}=45)$;

- Phase 4 (mixed period, from January to November 2019): cT1N0 patients treated using a uniportal approach while all other patients underwent a multiportal approach $(\mathrm{n}=26)$;

* Phase 5: (uniportal period, from December 2019 to August 2020): thoracoscopic lobectomy with ND2a-1/2 lymphadenectomy performed exclusively using a uniportal approach $(\mathrm{n}=27)$.

Figure 2 summarizes the patients included in each phase. Operative time, the rate of intraoperative massive bleeding, and the rate of postoperative prolonged air leak (PAL) were compared among the five phases. Figure 3 shows the operative time in all patients, and Figure 4 shows the operative time in patients undergoing the uniportal approach.

The 189 patients were also classified into two groups depending on the surgical approach, i.e., into a uniportal group ( $n=42$ ) and a multiportal group (group-1: $n=147$ ). The two groups were compared in terms of age, sex, tumor localization, histology and pathological stage, and perioperative results, including operative time, intraoperative blood loss, duration of postoperative drainage, rate of postoperative PAL (continuous air leak for $\geq 5$ days postoperatively), length of postoperative hospital stay, rate of conversion to thoracotomy, rate of intraoperative massive bleeding (bleeding requiring $>30 \mathrm{~s}$ of compression for hemostasis), rate of readmission within 30 days 
Table 1 Operative time, rate of postoperative PAL, and rate of intraoperative massive bleeding: comparison among the five phases of the study

\begin{tabular}{lcccccc}
\hline Variables & Phase 1 $(\mathrm{n}=45)$ & Phase $2(\mathrm{n}=46)$ & Phase 3 $(\mathrm{n}=45)$ & Phase $4(\mathrm{n}=26)$ & Phase $5(\mathrm{n}=27)$ & $\mathrm{P}$ value \\
\hline Operative time $(\mathrm{min})$ & $234 \pm 46$ & $222 \pm 58$ & $198 \pm 36$ & $166 \pm 57$ & $148 \pm 34$ & $<0.0001^{*}$ \\
PAL, $\mathrm{n}(\%)$ & $13(28.9)$ & $10(21.7)$ & $6(13.3)$ & $2(7.7)$ & $0(0)$ & $0.0061^{*}$ \\
Intraoperative massive bleeding, $\mathrm{n}(\%)$ & $4(8.9)$ & $2(4.3)$ & $1(2.2)$ & $0(0)$ & $1(3.7)$ & 0.5 \\
\hline
\end{tabular}

${ }^{*}$, significant values. PAL, prolonged air leak.

after discharge, morbidity (Clavien-Dindo grade $\geq$ III), and 30- and 90-day postoperative mortality. To address the imbalance in sample size and potential intrinsic differences between the two groups, the patients' characteristics were matched based on the propensity score, resulting in a uniportal group and a multiportal group-2, each with 42 patients. The same perioperative results described above were compared between these two groups.

\section{Surgical procedures}

In the uniportal approach to thoracoscopic lobectomy, a single 35-40-mm skin incision was made at the anterior axial line of the 4th or 5th intercostal space, and initially covered using an extra-small wound retractor (Alexis Wound Retractor; Applied Medical, Rancho Santa Margarita, CA, USA). The multiportal approach involved the use of three or four ports: a rigid port for the thoracoscope and two or three other ports for forceps or staples, which were covered using an extra-extra-small wound retractor.

Both types of surgery were performed with the patient under general anesthesia and receiving single-lung ventilation while in the lateral decubitus position. The surgical maneuvers were the same in both approaches. Dominant vessels, including the pulmonary artery and vein, were exposed sufficiently and then divided, mainly using endovascular staplers. Small branches of these vessels were divided using an energy device after proximal ligation with silk suture. The dominant bronchus was also divided using a stapler. Interlobar fissures were divided using an energy device and ligation with silk suture or staplers. After these procedures, the specimen was placed into a plastic bag and removed from the thorax. ND2a-1/2 lymphadenectomy was then performed. ND2a-1 lymphadenectomy means selective mediastinal dissection while ND2a-2 does radical mediastinal dissection (6). At the end of surgery, a chest tube for thoracic drainage was placed in the thorax.

\section{Statistical analysis}

The operative time, rate of intraoperative massive bleeding, and rate of postoperative PAL were compared among the surgical periods using the Welch test or Fisher's exact test. The relationship between operative time and the number of surgical experiences was assessed using Spearman's rank correlation test. The patient characteristics and perioperative results of the uniportal group were compared either with those of multiportal group-1 or multiportal group-2 using an independent $t$-test or Fisher's exact test. Differences were considered significant at $\mathrm{P}<0.05$. All calculations and statistical tests were performed using the EZR graphical user interface (Saitama Medical Centre, Jichi Medical University, Saitama, Japan).

\section{Results}

The operative time, rate of intraoperative massive bleeding, and rate of postoperative PAL across were compared among the five phases in Table 1. The operative time (phase 1: $234 \pm 46 \mathrm{~min}$, phase $2: 222 \pm 58 \mathrm{~min}$, phase $3: 198 \pm 36 \mathrm{~min}$, phase 4: $166 \pm 57 \mathrm{~min}$, phase 5: $148 \pm 34 \mathrm{~min}, \mathrm{P}<0.0001$ ) and rate of postoperative PAL (phase 1: $28.9 \%$, phase $2: 21.7 \%$, phase $3: 13.3 \%$, phase $4: 7.7 \%$, phase $5: 0 \%, \mathrm{P}=0.0061$ ) were significantly reduced from phase 1 to 5 . The rate of intraoperative massive bleeding did not change significantly (phase 1: $8.9 \%$, phase 2: $4.3 \%$, phase $3: 2.2 \%$, phase $4: 0 \%$, phase $5: 3.7 \%, \mathrm{P}=0.5$ ). The learning curve for the operative time in all consecutive cases $(\mathrm{n}=189)$ was shown in Figure 3. The significant correlation between operative time and the number of consecutive thoracoscopic major pulmonary resections with ND2a-1/2 lymphadenectomy ( $r=-0.579$, $\mathrm{P}<0.0001)$ indicated an improvement in operative time with increasing experience, despite the transition from a uniportal to a multiportal approach. Figure 4 showed the learning curve of the operative time for the 42 cases in which the uniportal approach was employed. The lack of 


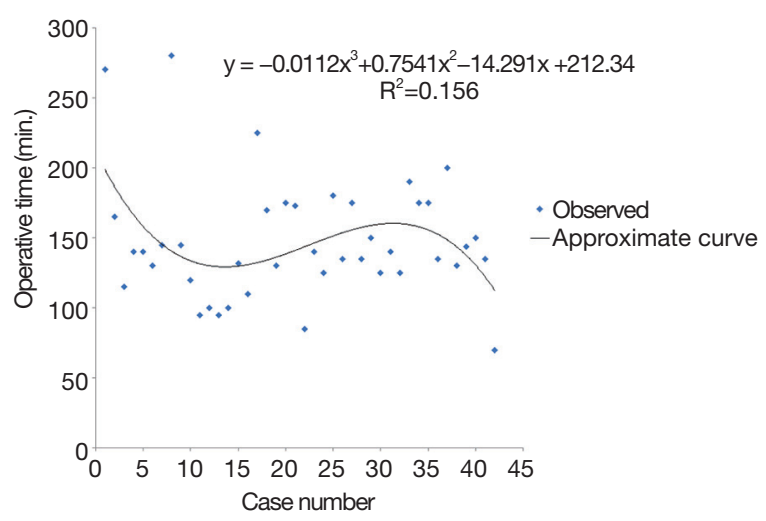

Figure 4 Assessment of the correlation between the operative time and number for consecutive cases $(n=42)$ in which a uniportal approach was used.

a significant correlation between operative time and the number of consecutive surgeries $(\mathrm{r}=0.06, \mathrm{P}=0.71)$ indicated the stability of the operative time.

Table 2 summarized the patient characteristics and perioperative results of the uniportal group, multiportal group-1 (before matching), and multiportal group-2 (after matching). Superior results were obtained in the uniportal versus multiportal group-1 with respect to the operative time $(147 \pm 43$ vs. $217 \pm 49$ min, respectively; $\mathrm{P}<0.0001)$, duration of postoperative drainage $(1.5 \pm 1.2$ vs. $3.1 \pm 2.1 \mathrm{~min}$, respectively; $\mathrm{P}<0.0001)$, and rate of postoperative $\mathrm{PAL}(2.4 \%$ vs. $20.4 \%$, respectively; $\mathrm{P}=0.0038$ ). The two groups did not significantly differ for any other perioperative parameter, including the rate of intraoperative massive bleeding $(2.4 \%$ vs. $4.8 \%$, respectively; $\mathrm{P}=0.69$ ). There was no 30 - or 90 day mortality in either the uniportal or multiportal-1 group. The comparison between the uniportal group and multiportal group-2 also showed superior results in terms of the operative time $(147 \pm 43$ vs. $207 \pm 50 \mathrm{~min}$, respectively; $\mathrm{P}<0.0001)$, duration of postoperative drainage $(1.5 \pm 1.2 v s$. $3.2 \pm 2 \mathrm{~min}$, respectively; $\mathrm{P}<0.0001)$, and rate of postoperative PAL (2.4\% vs. $26.2 \%$, respectively; $\mathrm{P}=0.0034)$, but there was no significant difference in any other perioperative parameter, including the rate of intraoperative massive bleeding.

\section{Discussion}

This report described one surgeon's (the author: HI) experience of thoracoscopic lobectomy with ND2a-1/2 lymphadenectomy, performed in patients with lung cancer using a multiportal or uniportal approach. The operative time, rate of postoperative PAL, and rate of intraoperative massive bleeding were assessed. While several reports have examined the learning curve in multiportal or uniportal thoracoscopic major pulmonary resection $(7-14)$, this is the first report in which the transition period from the traditional to less invasive procedure was considered.

Many studies have used the operative time to represent the surgical learning curve (7-15). Most of these studies revealed the improvement of operative time in line with gaining the surgical experiences although the surgical approach was different in each study. In this study, the operative time in all cases showed an improvement with increasing experience, and no decline after the introduction of the uniportal approach. This result indicated that operative time was not negatively affected by the introduction of a uniportal approach by using appropriate surgical techniques. On the contrary, the operative time in the cases receiving uniportal approach did not show an improvement with increasing experience during the study period. This might have been due to the small number of cases. In the study of Vieira et al., cubic spline analysis indicated proficiency after an initial phase of 60 cases and transition phase of 80 cases (8). Therefore, over time, increasing experience with the uniportal approach may result in an improved operative time.

In this study, the rate of postoperative PAL was taken to represent the learning curve for thoracoscopic lobectomy with ND2a-1/2 lymphadenectomy, because PAL can cause further pulmonary complications or negatively affect the postoperative recovery $(16,17)$. In the series of Attaar et al., pulmonary complications, 30-day readmission, and postoperative hospitalization were attributed to PAL (16). A reduction in the rate of postoperative PAL will not only avoid these complications, but will also contribute to a reduction in medical costs. However, other reports describing the learning curve of thoracoscopic surgery found no improvement in postoperative PAL (7-15). The difference may be due to the more sophisticated surgical techniques used during thoracoscopic surgery to dissect the fissure and thus expose the pulmonary artery or suture an air leak site. Alternatively, it may reflect the decision to use a fissureless technique in patients with a dense fissure. Several procedures have been introduced to reduce the rate of postoperative PAL, and at our hospital use of the fissureless technique during thoracoscopic lobectomy achieved this $(18,19)$. However, a highly experienced surgeon is required because the surgical steps are different from those of the 
Table 2 Comparison of patient characteristics and perioperative results between the uniportal and multiportal approach groups

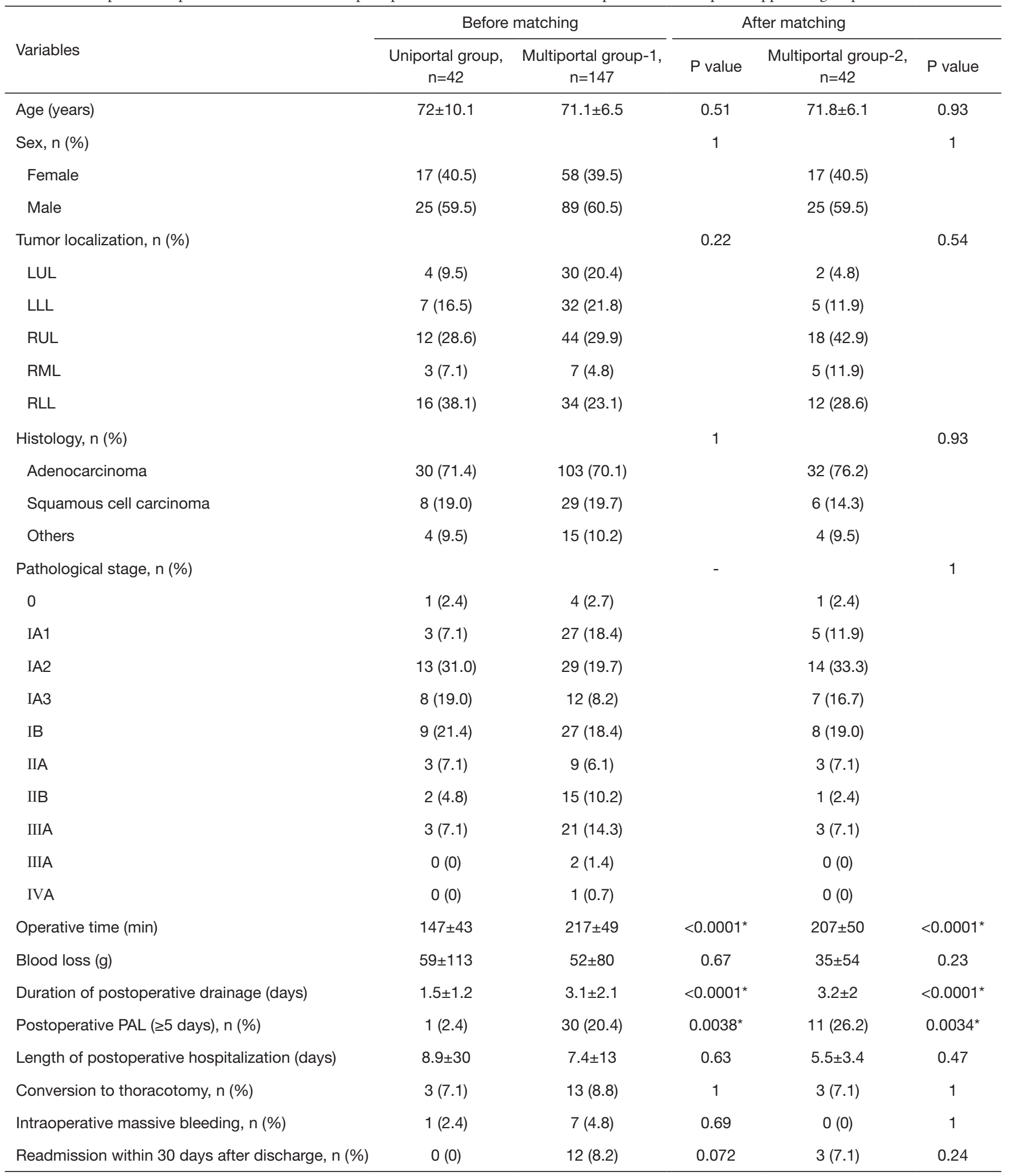

Table 2 (coninued) 
Table 2 (coninued)

\begin{tabular}{|c|c|c|c|c|c|}
\hline Variables & \multicolumn{2}{|c|}{ Before matching } & \multicolumn{2}{|c|}{ After matching } & $P$ value \\
\hline 30-day mortality & $0(0)$ & $0(0)$ & - & $0(0)$ & - \\
\hline
\end{tabular}

*, significant values. PAL, prolonged air leak; LLL, left lower lobe; LUL, left upper lobe; RLL, right lower lobe; RML, right middle lobe; RUL, right upper lobe.

conventional technique, in which the pulmonary arteries are dissected and exposed at the fissure. The improvement in the rate of postoperative PAL suggests that, with increasing experience, the fissureless technique was successfully implemented even for patients with a dense fissure.

The rate of intraoperative massive bleeding remained relatively stable over the five phases in this study, whereas both the operative time and the rate of postoperative PAL improved with increasing experience. A team from our hospital previously reported no difference in the rate of significant intraoperative bleeding during thoracoscopic anatomic pulmonary resection between the early and late phases (20). In their retrospective analysis, Yamashita et al. similarly described that increased experience did not reduce the risk of intraoperative massive bleeding during thoracoscopic surgery (21). Those results highlighted that the risk of intraoperative massive bleeding in patients undergoing thoracoscopic major pulmonary resection should be a concern even among highly experienced surgeons.

A comparison of the perioperative results obtained with the uniportal and multiportal approaches showed that those of the former approach were superior, including in terms of the operative time, duration of postoperative drainage, and rate of PAL, consistent with previous reports comparing uniportal and multiportal approaches (3-5). In addition to the improved operative time and postoperative PAL across the five consecutive phases in this study, the switch to a uniportal approach, despite its technical difficulties, did not affect the thoracoscopic surgery learning curve. Previous studies from our hospital described several surgical techniques suitable for uniportal thoracoscopic major pulmonary resections (22). Their adoption will promote continued improvement in the results achieved using the uniportal approach.

\section{Limitations}

The limitations of this study included its retrospective, single-center design and relatively small number of patients. In addition, although the patients' characteristics were matched based on the propensity score to address the imbalance in sample size and potential intrinsic differences between the two groups, the data were lack of other variables including body mass index or preoperative comorbidities which might affect the perioperative results. However, all surgeries were performed by a single surgeon (HI), such that the results reflect a "real" surgical learning curve. Moreover, we would like to insist that this manuscript described the learning curve of a single thoracic surgeon and successful implementation of uniportal approach, which was different from the superiority of uniportal approach compared to multiportal approach.

\section{Conclusions}

This study examined the learning curve of thoracoscopic lobectomy with ND2a-1/2 lymphadenectomy, between the first and fifth (final) phases and including the transition from a uniportal to a multiportal approach. The results indicated that the learning curve was not negatively affected by the introduction of a uniportal approach. The technical difficulties associated with this approach can be overcome by adopting a suitable surgical procedure.

\section{Acknowledgments}

The authors thanks all of the surgeons and coworkers who 
contributed to this study, as well as the editors and reviewers for their assistance with the manuscript.

Funding: None.

\section{Footnote}

Reporting Checklist: The authors have completed the STROBE reporting checklist. Available at https://dx.doi. org/10.21037/jtd-21-500

Data Sharing Statement: Available at https://dx.doi. org/10.21037/jtd-21-500

Peer Review File: Available at https://dx.doi.org/10.21037/ jtd-21-500

Conflicts of Interest: All authors have completed the ICMJE uniform disclosure form (available at https://dx.doi. org/10.21037/jtd-21-500). HI served as unpaid editorial board member of Fournal of Thoracic Disease from Aug 2020 to Jul 2022. The other authors have no conflict of interest to declare.

Ethical Statement: The authors are accountable for all aspects of the work in ensuring that questions related to the accuracy or integrity of any part of the work are appropriately investigated and resolved. The study was conducted in accordance with the Declaration of Helsinki (as revised in 2013). The study was approved by institutional ethics board of Japanese Red Cross Maebashi Hospital (NO.: 2021-13) and individual consent for this retrospective analysis was waived.

Open Access Statement: This is an Open Access article distributed in accordance with the Creative Commons Attribution-NonCommercial-NoDerivs 4.0 International License (CC BY-NC-ND 4.0), which permits the noncommercial replication and distribution of the article with the strict proviso that no changes or edits are made and the original work is properly cited (including links to both the formal publication through the relevant DOI and the license). See: https://creativecommons.org/licenses/by-nc-nd/4.0/.

\section{References}

1. Gonzalez D, Paradela M, Garcia J, et al. Single-port videoassisted thoracoscopic lobectomy. Interact Cardiovasc Thorac Surg 2011;12:514-5.
2. Rocco G, Martin-Ucar A, Passera E. Uniportal VATS wedge pulmonary resections. Ann Thorac Surg 2004;77:726-8.

3. Al-Ameri M, Sachs E, Sartipy U, et al. Uniportal versus multiportal video-assisted thoracic surgery for lung cancer. J Thorac Dis 2019;11:5152-61.

4. Bourdages-Pageau E, Vieira A, Lacasse Y, et al. Outcomes of uniportal vs multiportal video-assisted thoracoscopic lobectomy. Semin Thorac Cardiovasc Surg 2020;32:145-51.

5. Hirai K, Takeuchi S, Usuda J. Single-incision thoracoscopic surgery and conventional video-assisted thoracoscopic surgery: a retrospective comparative study of perioperative clinical outcomes. Eur J Cardiothorac Surg 2016;49:i37-41.

6. Okada M, Sakamoto T, Yuki T, et al. Selective mediastinal lymphadenectomy for clinic-surgical stage I non-small cell lung cancer. Ann Thorac Surg 2006;81:1028-32.

7. Hamada A, Oizumi H, Kato H, et al. Learning curve for port-access thoracoscopic anatomic lung segmentectomy. J Thorac Cardiovasc Surg 2018;156:1995-2003.

8. Vieira A, Bourdages-Pageau E, Kenny K, et al. The learning curve on uniportal video-assisted thoracic surgery: An analysis of proficiency. J Thorac Cardiovasc Surg 2020;159:2487-95.e2.

9. Duan L, Jiang G, Yang Y. One hundred and fiftysix cases of anatomical pulmonary segmentectomy by uniportal video-assisted thoracic surgery: a 2-year learning experience. Eur J Cardiothorac Surg 2018;54:677-82.

10. Bedetti B, Bertolaccini L, Solli P, et al. Learning curve and established phase for uniportal VATS lobectomies: the Papworth experience. J Thorac Dis 2017;9:138-42.

11. Stamenovic D, Messerrschmidt A, Schneider T. Cumulative sum analysis of the learning curve for uniportal video-assisted thoracoscopic lobectomy and lymphadenectomy. J Laparoendosc Adv Surg Tech A 2019;29:914-20.

12. Hernandez-Arenas LA, Lin L, Purmessur RD, et al. Uniportal video-assisted thoracoscopic early learning curve for major lung resections in a high volume training center. J Thorac Dis 2018;10:S3670-7.

13. Liu X, Chen X, Shen Y, et al. Learning curve for uniportal video-assisted thoracoscopic surgery lobectomy-results from 120 consecutive patients. J Thorac Dis 2018;10:5100-7.

14. Nachira D, Meacci E, Porziella V, et al. Learning curve of uniportal video-assisted lobectomy: analysis of 15-month experience in a single center. J Thorac Dis 
2018;10:S3662-9.

15. Arnold BN, Thomas DC, Bhatnagar V et al. Defining the learning curve in robot-assisted thoracoscopic lobectomy. Surgery 2019;165:450-4.

16. Attaar A, Luketich JD, Schuchert MJ, et al. Prolonged air leak after pulmonary resection increases risk of noncardiac complications, readmission, and delayed hospital discharge: A propensity score-adjusted analysis. Ann Surg 2021;273:163-72.

17. Konstantinidis K, Woodcock-Shaw J, Dinesh P, et al. Incidence and risk factors for 90-day hospital readmission following video-assisted thoracoscopic anatomical lung resection. Eur J Cardiothorac Surg 2019;55:666-72.

18. Igai H, Kamiyoshihara M, Yoshikawa R, et al. The efficacy of thoracoscopic fissureless lobectomy in patients with dense fissures. J Thorac Dis 2016;8:3691-6.

19. Igai H, Kamiyoshihara M. Thoracoscopic fissureless left

Cite this article as: Igai $\mathrm{H}$, Kamiyoshihara M, Furusawa S, Ohsawa F, Yazawa T, Matsuura N. The learning curve of thoracoscopic surgery in a single surgeon and successful implementation of uniportal approach. J Thorac Dis 2021;13(7):4063-4071. doi: 10.21037/jtd-21-500 upper lobectomy for the patient with a dense fissure. Multimed Man Cardiothorac Surg 2019;2019. doi: 10.1510/mmcts.2019.024.

20. Igai H, Kamiyoshihara M, Yoshikawa R, et al. Algorithmbased troubleshooting to manage bleeding during thoracoscopic anatomic pulmonary resection. J Thorac Dis 2019; 11:4544-50.

21. Yamashita S, Tokuishi K, Moroga T, et al. Totally thoracoscopic surgery and troubleshooting for bleeding in non-small cell lung cancer. Ann Thorac Surg 2013;95:994-9.

22. Igai H, Matsuura N, Kamiyoshihara M. Uniportal thoracoscopic upper division segmentectomy of left upper lobe using a unidirectional anterior approach. Multimed Man Cardiothorac Surg 2020;2020. doi: 10.1510/ mmcts.2020.017. 